\title{
Human Mesenchymal Stromal Cells Decrease Mortality Following Intestinal Ischemia and Reperfusion Injury
}

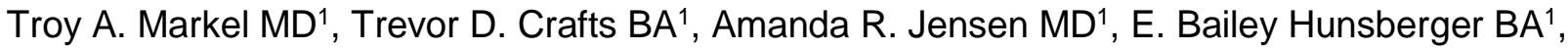 \\ and Mervin C. Yoder MD² \\ ${ }^{1}$ Department of Surgery, Section of Pediatric Surgery \\ ${ }^{2}$ Department of Pediatrics, Section of Neonatology \\ Riley Hospital for Children at Indiana University Health and \\ The Indiana University School of Medicine \\ Indianapolis, IN
}

TAM performed survival ischemia/reperfusion experiments, performed statistical analysis, wrote the manuscript, edited the manuscript for final content; TDC performed 6 hour reperfusion ischemia/reperfusion experiments, assisted in writing manuscript, edited the manuscript for final content; ARJ performed cell culture experiments, edited manuscript for final content; EBH performed histology experiments, cytokine assays, and edited the manuscript for final content; MCY provided critical advisement and insight for the project, edited the manuscript for final content.

No disclosures to report

Correspondence:

Troy A. Markel

Assistant Professor of Surgery

Indiana University School of Medicine

Riley Hospital for Children at IU Health

705 Riley Hospital Dr.

RI 2500

Indianapolis, IN 46202

317-437-2506

This is the author's manuscript of the article published in final edited form as:

Markel, T. A., Crafts, T. D., Jensen, A. R., Hunsberger, E. B., \& Yoder, M. C. (2015). Human mesenchymal stromal cells decrease mortality after intestinal ischemia and reperfusion injury. Journal of Surgical Research, 199(1), 56-66. http://doi.org/10.1016/j.jss.2015.06.060 


\section{ABSTRACT}

Background: Cellular therapy is a novel treatment option for intestinal ischemia. Bone Marrow Derived Mesenchymal Stromal Cells (BMSCs) have previously been shown to abate the damage caused by intestinal ischemia/reperfusion injury (I/R). We therefore hypothesized that 1) human BMSCs (hBMSCs) would produce more beneficial growth factors and lower levels of proinflammatory mediators compared to differentiated cells, 2) direct application of hBMSCs to ischemic intestine would decrease mortality following injury, and 3) decreased mortality would be associated with an altered intestinal and hepatic inflammatory response.

Methods: Adult hBMSCs and keratinocytes were cultured on polystyrene flasks. For in vitro experiments, cells were exposed to TNF, LPS, or 2\% oxygen for twenty-four hours.

Supernatants were then analyzed for growth factors and chemokines by multiplex assay. For in vivo experiments, eight to twelve week old male C57BI6J mice were anesthetized and underwent a midline laparotomy. Experimental groups were exposed to temporary superior mesenteric artery occlusion for 60 minutes. Immediately following ischemia, $2 \times 10^{6} \mathrm{hBMSCs}$ or keratinocytes in PBS were placed into the peritoneal cavity. Animals were then closed and allowed to recover for 6 hours (molecular / histological analysis) or 7 days (survival analysis). Following 6 hour reperfusion, animals were euthanized. Intestines and livers were harvested and analyzed for inflammatory chemokines, growth factors, and histological changes.

Results: hBMSCs expressed higher levels of human IL-6, IL-8, VEGF, and EGF, and lower levels of IL1, IL3, IL7, and GMCSF following stimulation. In vivo, I/R resulted in significant mortality (70\% mortality), while application of hBMSCs following ischemia decreased mortality to $10 \%$ in a dose dependent fashion $(p=0.004)$. Keratinocyte therapy offered no improvements in mortality above I/R. Histological profiles were equivalent between ischemic groups, regardless of application of hBMSCs or keratinocytes. Cellular therapy yielded significantly decreased 
murine intestinal levels of SALK-1, betacellulin and endothelin, while increasing levels of eotaxin, MIG, MCP-1, IL-6, GCSF and IP-10 from ischemia were appreciated. hBMSC therapy yielded significantly higher expression of murine intestinal VEGF and lower levels of intestinal MIG compared to keratinocyte therapy. Application of hBMSCs following ischemia yielded significantly lower murine levels of hepatic MIG, IP-10, and GCSF compared to keratinocyte therapy.

Conclusion: Human BMSCs produce multiple beneficial growth factors. Direct application of hBMSCs to the peritoneal cavity following intestinal I/R decreased mortality by sixty percent. Improved outcomes with hBMSC therapy were not associated with improved histological profiles in this model. hBMSC therapy was associated with higher VEGF in intestines, and lower levels of proinflammtory MIG, IP-10, and GCSF in liver tissue following ischemia, suggesting that reperfusion with hBMSC therapy may alter survival by modulating the systemic inflammatory response to ischemia.

\section{INTRODUCTION}

Intestinal ischemia and necrosis affect multiple patient populations of varying ages and comorbidities. Acute mesenteric ischemia (AMI) is prevalent in the elderly population and those who undergo cardiac bypass surgery. AMI affects nearly 5000 patients annually, with many requiring open or endovascular surgical intervention to lyse the clot and salvage the ischemic intestine. The mortality rate for AMI can be as high as $40 \%$ for those who progress to surgery (1). Necrotizing enterocolitis and volvulus are two forms of intestinal ischemia that can affect the neonatal population. Necrotizing enterocolitis, which has multiple factors contributing to its etiology, affects the very low birth weight premature population. The mortality for the most severe cases of NEC can be quite high (2). Midgut volvulus associated with malrotation occurs much less frequently than NEC, but carries a high mortality when a majority of the bowel is 
involved (3). In either case, if patients survive these ischemic episodes, they often require prolonged hospitalization and long term parenteral nutrition.

Significant elevations in serum chemokines, including monocyte chemoattractant protein 1 (MCP-1), eotaxin, and chemokine ligand 10 (IP-10) have been noted following intestinal ischemia, and are thought to be responsible for leukocyte mobilization to the areas of injured bowel $(4,5)$. These cells are responsible for injury repair, but also promote inflammation, which may be detrimental to the host. For example, lymphocyte influx is thought to be detrimental to recovery, as lymphocyte depleted animals had better outcomes following intestinal ischemia (6). However, other leukocyte classes may actually promote intestinal recovery by digesting dead cells and repairing the extracellular matrix (7). Additionally, markers of neovascularization have been elevated after intestinal ischemia $(8,9)$. Neovasculogenesis increases intestinal capillary density to restore oxygen balance and nutrient homeostasis to injured bowel.

In this regard, stem cells have become an increasingly plausible mode of therapy for ischemic tissues, mainly due to their immunogenic and angiogenic reparative properties (10-12). Bone marrow mesenchymal stromal cells (BMSCs) have been shown to ameliorate the destructive effects seen during intestinal ischemia, and may serve as a novel treatment option for this condition. BMSCs work to decrease intestinal permeability and to promote recovery of the gut-mucosal barrier following injury $(13,14)$. Human bone marrow mesenchymal stromal cells (hBMSCs) have not been previously assessed in preclinical intestinal ischemic models. We therefore hypothesized that 1) human BMSCs would produce more beneficial growth factors and lower levels of proinflammatory chemokines compared to differentiated cells, 2) direct application of hBMSCs to ischemic intestine would decrease mortality following injury in a dose dependent fashion, and 3) decreased mortality would be associated with altered post-ischemic intestinal and hepatic inflammation. 


\section{METHODS}

\section{Cell Culture}

hBMSCs were obtained from Dr. Darwin Prokop at Texas A\&M University. His lab is NIH funded to procure, purify, and verify hBMSCs from human subjects. Cells from two donors were obtained and plated separately onto $75 \mathrm{~cm}^{2}$ polystyrene flasks. Cells were cultured in alpha-MEM (Life Technologies) with 16\% FBS (Atlanta Biologicals), 1\% Glutamine and 1\% pen/strep (Sigma) at $37 \mathrm{C}$ in $5 \% \mathrm{CO}_{2}$ in air. Once cells reached $90 \%$ confluence, they were passaged by adding TrypLE Express (Life Technologies) to the culture flask. Cells were used between passages 2 and 10 .

Human nTERT keratinocytes were graciously donated by Dr. Jeffery Travers at the Indiana University School of Medicine. Cells were originally purchased through ATCC and were cultured in Epilife media with keratinocyte growth factor (Life Technologies) at $37 \mathrm{C}$ in $5 \% \mathrm{CO}_{2}$ in air. Once cells reached 90\% confluence, they were passaged by adding TrypLE Express (Life Technologies) to the culture flask. Cells were used between passages 24 and 35.

\section{In Vitro Experiments}

Once ready for experimentation, cells were lifted from their flasks with TrypLE Express as above. Cells were then pelleted at $400 \mathrm{~g}$ for $5 \mathrm{mins}$ and resuspended in fresh media. Cells were then counted with the aid of an automated fluorescent cell (Luna-FL Automated Cell Counter, Logos Biosystems). One hundred thousand hBMSCs or keratinocytes were plated into each well of a 12 well plate and allowed to adhere to the plastic overnight. Media was changed the next day and cells were exposed to one of three noxious stimuli for 24 hours: 1) TNF $50 \mathrm{ng} / \mathrm{ml}(15), 2)$ LPS $200 \mathrm{ng} / \mathrm{ml}$ (15), or 3) 24 hours of hypoxia with $2 \%$ oxygen. Supernatants were collected and stored at $-20 \mathrm{C}$ until analysis. 


\section{Murine Ischemia/Reperfusion Model}

The experimental protocol and use of animals was approved by the Indiana University Institutional Animal Care and Use Committee. Adult male C57BI6J mice (8-12 weeks, 20-30g, Jackson Labs) were allowed to acclimate to their environments for at least 48 hours prior to intervention. They had free access to standard chow and were maintained in a $12 \mathrm{~h}$ light-dark cycle. Mice were anesthetized with $3 \%$ isoflurane and maintained at $1 \%$ isoflurane for the duration of the procedure. Abdomens were then shaved and prepped with $70 \%$ ethanol and betadine. One milliliter of $0.9 \%$ normal saline was injected subcutaneously and a midline laparotomy performed. The intestines were eviscerated and the small bowel mesenteric root was identified.

In ischemic groups, the mesenteric root was temporarily occluded with an atraumatic micro-vascular clamp for 60 minutes. During the period of ischemia, the abdomen was temporarily closed with silk suture to prevent evaporative heat losses. Animals were maintained on a heating blanket to maintain body temperature. After 60 minutes, abdomens were reopened and the clamp was removed. The abdominal fascia and skin were then closed in two layers. Prior to final closure of the facial defect, hBMSCs, keratinocytes, or 250ul of PBS vehicle were applied directly into the peritoneal cavity. A dose response curve was performed and 2 million hBMSCs (or keratinocytes) in 250ul of PBS were chosen for the remainder of experiments because they yielded the best survival advantage (Figure 3). Following abdominal closure, triple antibiotic ointment was applied to the incision and analgesia (1 mg/kg buprenorphine and $5 \mathrm{mg} / \mathrm{kg}$ caprofen) was injected subcutaneously. Animals were then allowed to awaken from anesthesia, were placed back in their cage, and were returned to animal housing.

\section{Survival Analysis}


Animals designated for survival analysis ( $N=5$ sham, 10 l/R, 20 I/R + hBMSC, 10 I/R + keratinocyte) were monitored twice daily over 7 days following the surgery for death, pain, and incisional complications. End points of analysis included animal death or when Laboratory Animal Resource Center veterinarians felt that animals were suffering and needed to be euthanized. Survival curves were then created based on these endpoints.

\section{$\underline{\text { Histology }}$}

After 6 hours of reperfusion, animals were euthanized and intestinal segments approximately $2 \mathrm{~cm}$ from the cecum were harvested (N=6 sham, 6 I/R, 12 I/R + hBMSC, 6 I/R + keratinocytes). Segments were placed into $4 \%$ paraformaldehyde and subsequently dehydrated in $70 \%$ ethanol. Segments were then paraffin embedded, and cut with a microtome. Tissue segments were placed on slides and were stained with H\&E. Histologic scoring of the depth of tissue injury was performed as described by Watkins, et al.: 0 , no damage; 1 , subepithelial space at the villous tip; 2 , loss of mucosal lining of the villous tip; 3 , loss of less than half of the villous structure; 4 , loss of more than half of the villous structure; 5 , transmural necrosis (11).

\section{Analysis of Supernatant and Tissue Cytokines}

Human IL-1, IL-3, IL-6, IL-7, IL-8, vascular endothelial growth factor (VEGF), epidermal growth factor (EGF), and granulocyte-monocyte colony stimulating factor (GMCSF) in cell supernatants were quantified with a Bioplex 200 multiplex beaded assay system (Bio-Rad) using multiplex plates for human inflammatory chemokines and growth factors (Millipore). Assays were performed at 1:1 dilution according to the manufacturer's instructions ( $N=4-6$ from 2-3 separate experiments).

Mouse intestinal and hepatic tissue segments ( $N=6$ sham, 6 I/R, 12 I/R + hBMSC, 6 I/R + keratinocytes) were thawed and homogenized in RIPA buffer (Sigma) with protease and 
phosphatase cocktail inhibitors (1:100 dilution, Sigma). Homogenates were centrifuged at $12,000 \mathrm{rpm}$ to pellet extraneous tissue, and supernatants were transferred to fresh eppendorff tubes for storage at -80C. Total protein concentration was then quantified by Bradford Assay using a spectrophotometer (Versamax microplate reader, Molecular Devices). Murine expression of soluble activin receptor-like kinase 1 (sALK-1), betacellulin, endothelin, VEGF, IL6, IP-10, eotaxin, monokine induced by gamma interferon (MIG), and MCP-1 were quantified with a Bioplex 200 multiplex beaded assay system (Bio-Rad) using multiplex plates for murine inflammatory chemokines and growth factors (Millipore). Assays were performed at 1:25 dilution according to the manufacturer's instructions.

\section{$\underline{\text { Statistical Analysis }}$}

Data are expressed as the mean $+/$ - the standard error of the mean. Survival was compared using the Mantel-Cox log rank test and the Gehan-Breslow-Wilcoxon test. Students t test was performed to compare groups for cytokine analysis. A p value of less than 0.05 was considered statistically significant.

\section{RESULTS}

hMSCs produce high levels of growth factors and low levels of proinflammatory chemokines

hBMSCs produced higher levels of VEGF and EGF at baseline and after stimulation with TNF, LPS, and hypoxia compared to their keratinocyte counterparts. IL-6 was also higher in TNF stimulated hBMSCs, and IL-8 higher in TNF and LPS stimulated hBMSCs compared to keratinocytes (Figure 1). In contrast, keratinocytes produced significantly higher levels of IL-1, IL-3, IL-7, and GMCSF at baseline and with stimulation as compared to hBMSCs (Figure 2). Thus, the profile of cytokine and chemokine secretion differed between hBMSCs and keratinocytes at baseline and following stimulation. 
$\underline{\text { hMSCS decrease mortality following intestinal I/R in a dose dependent manner }}$

Temporary occlusion of the superior mesenteric artery resulted in marked intestinal ischemia and a 7 day mortality rate of $70 \%$ compared to $0 \%$ mortality in sham animals (Figure $3 A, p=0.04)$. Addition of hBMSCs following ischemia significantly decreased the mortality in a dose dependent fashion (Figure 3B). Maximum benefit was seen with the application of 2 million hBMSCs, the highest dose tested. Application of 2 million hBMSCs following ischemia decreased the seven day mortality to 10 percent (Figure $3 C, p=0.0004$ ). No survival benefit was seen with infusion of human keratinocytes following intestinal I/R. These data identify hBMSCs as providing a robust survival advantage to mice following experimental intestinal I/R injury compared to human keratinocytes.

\section{$\underline{\text { hBMSCs do not improve histological profiles following } 6 \text { hour reperfusion }}$}

When assaying intestinal histology, sham animals maintained intact crypt/villus structures with normal appearing goblet cells and epithelium (Figure 4A). Intestinal morphology from ischemic animals was noted to have shortened crypt villus height, decreased goblet cells, and notable bowel wall hemorrhage in focal regions (Figure 4B). Cellular therapy with hBMSCs (Figure 4C) or keratinocytes (4D) demonstrated similar histologic profiles compared to I/R animals without therapy. Sham animals had an average histology score of $0,1 / R$ animals an average score of $0.83+/-0.83, \mathrm{I} / \mathrm{R}+\mathrm{hBMSC}$ animals an average score of $2.0+/-.59$, and I/R + keratinocytes an average of $1.5+/-0.67$ (Figure E). These results suggest that the survival benefits provided to mice following intestinal I/R injury by direct application of hBMSCs were not related to significant improvements in the morphology of the recovering intestine.

\section{Angiogenic growth factors and pro-inflammatory chemokines are altered with cellular therapy}

Intestinal I/R significantly increased murine intestinal tissue levels of SALK-1, endothelin, betacellulin, eotaxin, MCP-1, IP-10, and IL-6, but had little effect on VEGF, GCSF, and MIG. 
hBMSC and keratinocyte therapy following I/R significantly decreased tissue levels of SALK-1, endothelin, and betacellulin, but hBMSC therapy yielded significantly higher levels of intestinal VEGF compared to intestine from animals treated with keratinocytes (Figure 5). Cellular therapy also significantly increased murine intestinal levels of eotaxin, MIG, IP-10, and MCP-1, which are involved in leukocyte chemotaxis and inflammation (Figure 6). Murine intestinal IL-6 trended higher with hBMSC therapy, but did not reach statistical significance. Intestinal MIG was noted to be significantly higher in keratinocyte treated groups compared to hBMSC therapy.

Murine hepatic levels of measured angiogenic factors were not significantly altered by intestinal I/R without therapy (Figure 8). Murine hepatic levels of MIG, and GCSF were elevated by intestinal ischemia, and MIG, IP-10, and GCSF were significantly lower in hBMSC treated livers than in keratinocyte treated livers (Figure 9). These results suggested that local delivery of hBMSCs and keratinocytes into the peritoneal cavity of mice following intestinal I/R injury resulted in differential activation of liver chemokine and cytokine expression.

\section{DISCUSSION}

Intestinal ischemia and necrosis impact a wide variety of patient populations that include the very young to the very old. Current therapy for intestinal ischemia is lacking, and surgical therapy is limited to operative interventions that relieve the ischemia as in cases of mesenteric thrombus, volvulus, or bowel obstruction. Many times though, surgeons are forced to perform salvage operations where involved bowel is excised due to necrosis or perforation. Surgeons therefore have a unique opportunity to salvage ischemic intestine through the direct application of novel drugs, devices, or cellular therapies to the injured bowel at the time of surgical intervention. Alternative modes of therapy are clearly warranted to rescue injured bowel and increase survival both during and following ischemia. In this study, we found that human BMSCs decreased mortality following intestinal ischemia. We also appreciated that hBMSCs 
secreted growth factors and produced lower levels of proinflamatory mediators compared to keratinocytes. In addition hBMSCs triggered higher levels of murine intestinal VEGF production, and lower levels of hepatic proinflammatory chemokine production compared to animal treated with keratinocyte control cells.

Previous studies have demonstrated the benefits of BMSCs during ischemia and reperfusion injury (16-18). However this is the first study to demonstrate a survival advantage with the use of BMSCs for intestinal ischemic pathology. Additionally it is the first study to report the use of human BMSCs in this disease process. Although the exact cause of death was unclear following mesenteric occlusion, previous studies have denoted acute cardiovascular collapse (19), alveolo-capillary dysfunction, and debilitating pulmonary hypertension (20) as causative factors of mortality. Additionally, increased intestinal permeability and bacterial translocation likely lead to a state of sepsis and end organ failure. Intestinal perforation was not appreciated and did not appear to be a cause of death in this study.

Stromal cells likely provide their beneficial effects to injured tissues through a multifactorial approach. First, BMSCs may integrate into injured tissue, differentiate into end organ cells and work to restore tissue architecture (21). Although several studies have showed engraftment of these cells following transplantation (22) this scenario is not as likely in this model given that animals started to succumb in as little as 6-12 hours following intestinal injury. Integration, differentiation, and restitution would likely take longer to accomplish in order to salvage the animals from this time point. A second plausible method of protection is that the BMSC binds to an injured intestinal cell and "downloads" vital materials into the native cell such as mitochondria, ATP, or other vital nutrients $(23,24)$. Mitochondrial dysfunction and energy uncoupling are an underlying result of the disruption of oxygen and substrate supply to tissues during ischemia (25). This disruption, which can happen in as little as 15 minutes, can lead to sustained depolarization, electrical uncoupling, and cell death (25). This mode of protection is 
possible, as it could happen fairly quickly upon initiation of therapy. A third possible mechanism of therapy is through the release of paracrine mediators (26). This is highly plausible in that the hBMSCs could release multiple factors that then work both locally and systemically to mitigate the detrimental factors that are being released by the bowel during ischemia/reperfusion. This study demonstrates that hBMSCs release a number of beneficial factors, including VEGF and EGF, and lower levels of proinflammatory mediators including IL-1 and GMCSF.

Other studies have noted improved histological profiles after mesenchymal stromal cell therapy (11). It is not entirely clear why the histological profiles for those animals treated with cellular therapy were higher, albeit not significantly higher, than non-treated animals. Many studies that have examined BMSC reparative effects on the bowel have either limited the area of injury to the terminal ileum, or have injected the BMSCs directly into the submucosa, thereby localizing the area of injury, therapy, and study. Injury of the entire small bowel in our model along with injection of the cells into the entire peritoneal cavity may have effectively diluted the cells, thereby limiting the ability of the cells to promote histologically reparative effects on such a large scale. Perhaps even larger doses of cells or infusion by an intravenous route would have accomplished these changes. It is also possible that improved histology scores would have been appreciated with a longer reperfusion period, but we were interested in what the bowel would look like before animals started to die, which began to occur between 6 and 12 hours after injury. We therefore chose the 6 hour reperfusion period to capture all viable subjects.

Murine intestinal proinflammatory chemokines, including IL-6, IP-10, eotaxin, MIG, and MCP-1 were elevated in the intestine in animals receiving either hBMSC or keratinocyte therapy. These chemokines are markers of inflammation and would suggest an elevated intestinal stimulus for enhancing leukocyte chemotaxis and homing to the area of injury. Elevations in these factors corroborate the observed histology profiles in that cellular therapy 
seemed to increase the histological injury scores. Elevated leukocytes in the area of injury are likely necessary for repair of damaged tissues and removal of dead cells and debris.

Murine intestinal SALK-1, betacellulin and endothelin were all noted to be decreased in cell treated ischemic groups. These factors are involved in endothelial mobilization and neovasculogenesis $(27,28)$. It was initially expected that these markers might be elevated with hBMSC therapy, thereby indicating that the stromal cells promote survival by increasing neovasculogenesis. However, neoangiogenic markers were decreased, possibly suggesting a decreased need for new blood vessel formation. VEGF, a common chemokine involved in neovasculogenesis and tissue ischemia (29), was not observed to be elevated in ischemic intestinal tissue extracts or those treated with hBMSCs in this model. However, VEGF from murine intestines treated with hBMSCs was significantly higher than VEGF levels in keratinocyte treated groups. One reason for the lack of elevation with ischemia could be due to its relatively short half-life (29), and therefore, tissue levels may have normalized during the six hour reperfusion period.

Murine hepatic levels of angiogenic factors were not readily affected with the intestinal I/R model. Murine hepatic levels of the proinflammatory mediators MIG and GCSF were elevated by intestinal ischemia, and MIG, IP-10, and GCSF were significantly higher in keratinocyte treated livers than in hBMSC treated livers. Lower levels of these proinflammatory mediators in the liver following hBMSC therapy may suggest that hBMSCs work to decrease systemic inflammation, rather than acting locally at the sites of injury.

A potential reason for the discrepant differences seen with human cellular therapy may be due to the barriers and incomplete understandings associated with xenotransplantation. In theory, one should not be able to place human cells into a mouse model without the cells being destroyed by the host. However, mesenchymal stromal cells have unique immunomodulatory 
properties that suppress T-lymphocyte proliferation and allow them to be transplanted across species (30). There have been at least 27 different studies where human mesenchymal stromal cells have been placed into immunocompetent hosts of different species (31). Interestingly, in many of these cases, the cells survived several weeks and even engrafted into host tissue (22). Despite the perceived advantages of BMSC therapy, the mechanism for this immunosuppression is not completely understood, and some investigators have even appreciated persistent $C D 4^{+}$lymphocyte immune responses following xenogenic mesenchymal stromal cell transplant (32). These responses are often short lived though, and tend to diminish over time (33). However, as previously mentioned, the benefits of hBMSC therapy clearly come within the first 12-24 hours of therapy, as many animals exposed to intestinal I/R injury that were not treated with hBMSCs succumbed during this time period.

\section{CONCLUSION}

Human BMSCs have shown promise in the treatment of intestinal ischemic pathology. Herein, we demonstrated that hBMSCs secrete beneficial growth factors and limit inflammatory chemokine production following stimulation. Moreover, hBMSCs decrease mortality following intestinal ischemia and reperfusion injury as compared to animals treated with a differentiated keratinocyte control cell population. The mechanism of this survival benefit is likely multifactorial, but may be associated with a decreased systemic inflammatory response associated with hBMSC therapy. Although the release of paracrine mediators from hBMSCs is very plausible, additional studies are needed to define which mediators are most important. Further studies will focus on utilizing molecular techniques to silence beneficial paracrine factors more prominent in hBMSCs (VEGF and EGF) and detrimental factors in keratinocytes (IL-1, GCSF) in order to see if survival and tissue inflammation can be normalized. 


\section{FIGURE LEGENDS}

Figure 1: hBMSCs produce higher levels of (A) VEGF, (B) EGF, (C)IL-6, and (D) IL-8 compared to keratinocytes when stimulated with TNF, LPS and hypoxia. * $=p<0.05$ compared to keratinocyte counterpart

Figure 2: Keratinocytes produce more (A) IL-1, (B) IL-3, (C) IL-7, and (D) GMCSF compared to hBMSCs when stimulated with TNF, LPS, and hypoxia. * $=p<0.05$ compared to hBMSC counterpart

Figure 3: A) SMA occlusion resulted in 70\% seven day mortality, while (B) application of human BMSCs after ischemia resulted in decreased mortality in a dose dependent fashion. (C) Use of hBMSCs decreased 7 day mortality by 60 percent compared to no therapy or keratinocyte therapy. ${ }^{*}=p=0.04$ versus Sham, $\#=p=0.0004$ versus I/R

Figure 4: Representative H\&E stained intestine following (A) Sham laparotomy, (B) I/R injury, (C) I/R + hBMSC therapy, and (D) I/R + keratinocyte therapy. (E) Histology scoring of intestinal specimens. 0 , no damage; 1 , subepithelial space at the villous tip; 2 , loss of mucosal lining of the villous tip; 3 , loss of less than half of the villous structure; 4 , loss of more than half of the villous structre; 5, transmural necrosis.

Figure 5: Cellular therapy decreased intestinal tissue levels of (A) sALK-1, (B) betacellulin, and (C) endothelin. (D) Levels of VEGF were not altered by intestinal I/R or with the use of hBMSCs. Keratinocyte therapy also decreased levels of these factors. Murine intestinal VEGF levels were significantly decreased following keratinocyte therapy. ${ }^{*}=p<0.05$ versus sham, $\#=$ $p<0.05$ versus $I / R, \$=p<0.05$ versus $I / R+h B M S C s$.

Figure 6: Cellular therapy increased intestinal tissue levels of $(A)$ eotaxin, $(B)$ MIG, $(C)$ MCP-1, (D) IP-10, (E) IL-6, and (F) GCSF. Keratinocyte therapy yielded similar elevations in these 
factors, with the only significant difference being with MIG * $=p<0.05$ versus sham, $\#=p<0.05$ versus $\mathrm{I} / \mathrm{R}, \$=\mathrm{p}<0.05$ versus $\mathrm{l} / \mathrm{R}+\mathrm{hBMSC}$.

Figure 7: Intestinal ischemia did not have a significant effect on murine hepatic production of angiogenic factors. Hepatic levels of (A) SALK-1 were not detectable. (B) betacellulin, and (C) endothelin have similar levels between sham, I/R, and I/R + cellular therapy groups. (D) Murine hepatic levels of VEGF were not altered by intestinal I/R or with the use of hBMSCs. Keratinocyte therapy, however, significantly increased detectable stores of murine hepatic VEGF. $\quad *=p<0.05$ versus sham $\#=p<0.05$ versus $I / R$

Figure 8: Intestinal ischemia or cellular therapy did not have an effect on eotaxin, MCP1, or IL-6 levels. Cellular therapy increased hepatic tissue levels of (B) MIG and (F) GCSF. Keratinocyte therapy after I/R yielded significantly higher levels of (B) MIG, (D) IP-10, and (F) GCSF compared to hBMSC therapy * $=p<0.05$ versus sham, $\#=p<0.05$ versus $\mathrm{I} / \mathrm{R}, \$=p<0.05$ versus l/R + hBMSCs.

\section{ACKNOWLEDGEMENTS}

This project was supported by Indiana University Health, Indianapolis, IN 


\section{REFERENCES}

1. Paladino NC, Inviati A, Di Paola V et al. Predictive factors of mortality in patients with acute mesenteric ischemia. A retrospective study. Ann Ital Chir 2013;84.

2. Markel TA, Crisostomo PR, Wairiuko GM, Pitcher J, Tsai BM, Meldrum DR. Cytokines in necrotizing enterocolitis. Shock 2006;25:329-37.

3. Mehall JR, Chandler JC, Mehall RL, Jackson RJ, Wagner CW, Smith SD. Management of typical and atypical intestinal malrotation. Journal of pediatric surgery 2002;37:116972.

4. Santen S, Wang Y, Laschke MW, Menger MD, Jeppsson B, Thorlacius H. Rho-kinase signalling regulates $\mathrm{CXC}$ chemokine formation and leukocyte recruitment in colonic ischemia-reperfusion. Int J Colorectal Dis 2010;25:1063-70.

5. Jawa RS, Quist E, Boyer CW, Shostrom VK, Mercer DW. Mesenteric ischemiareperfusion injury up-regulates certain $\mathrm{CC}, \mathrm{CXC}$, and $\mathrm{XC}$ chemokines and results in multi-organ injury in a time-dependent manner. Eur Cytokine Netw 2013;24:148-56.

6. Chen J, Crispin JC, Tedder TF, Dalle Lucca J, Tsokos GC. B cells contribute to ischemia/reperfusion-mediated tissue injury. J Autoimmun 2009;32:195-200.

7. McCracken JM, Allen LA. Regulation of human neutrophil apoptosis and lifespan in health and disease. J Cell Death 2014;7:15-23.

8. El-Assal ON, Paddock H, Marquez A, Besner GE. Heparin-binding epidermal growth factor-like growth factor gene disruption is associated with delayed intestinal restitution, impaired angiogenesis, and poor survival after intestinal ischemia in mice. Journal of pediatric surgery 2008;43:1182-90.

9. Ishii M, Tanaka E, Imaizumi T et al. Local VEGF administration enhances healing of colonic anastomoses in a rabbit model. Eur Surg Res 2009;42:249-57. 
10. Mendez JJ, Ghaedi M, Sivarapatna A et al. Mesenchymal stromal cells form vascular tubes when placed in fibrin sealant and accelerate wound healing in vivo. Biomaterials 2015;40:61-71.

11. Watkins DJ, Yang J, Matthews MA, Besner GE. Synergistic effects of HB-EGF and mesenchymal stem cells in a murine model of intestinal ischemia/reperfusion injury. Journal of pediatric surgery 2013;48:1323-9.

12. Glenn JD, Whartenby KA. Mesenchymal stem cells: Emerging mechanisms of immunomodulation and therapy. World J Stem Cells 2014;6:526-39.

13. Jiang H, Qu L, Li Y et al. Bone marrow mesenchymal stem cells reduce intestinal ischemia/reperfusion injuries in rats. J Surg Res 2011;168:127-34.

14. Yang J, Watkins D, Chen CL, Bhushan B, Zhou Y, Besner GE. Heparin-binding epidermal growth factor-like growth factor and mesenchymal stem cells act synergistically to prevent experimental necrotizing enterocolitis. J Am Coll Surg 2012;215:534-45.

15. Crisostomo PR, Wang Y, Markel TA, Wang M, Lahm T, Meldrum DR. Human mesenchymal stem cells stimulated by TNF-alpha, LPS, or hypoxia produce growth factors by an NF kappa B- but not JNK-dependent mechanism. Am J Physiol Cell Physiol 2008;294:C675-82.

16. Dernbach E, Urbich C, Brandes RP, Hofmann WK, Zeiher AM, Dimmeler S. Antioxidative stress-associated genes in circulating progenitor cells: evidence for enhanced resistance against oxidative stress. Blood 2004;104:3591-7.

17. Crisostomo PR, Wang M, Herring CM et al. Gender differences in injury induced mesenchymal stem cell apoptosis and VEGF, TNF, IL-6 expression: role of the $55 \mathrm{kDa}$ TNF receptor (TNFR1). J Mol Cell Cardiol 2007;42:142-9. 
18. Markel TA, Wang Y, Herrmann JL et al. VEGF is critical for stem cell-mediated cardioprotection and a crucial paracrine factor for defining the age threshold in adult and neonatal stem cell function. Am J Physiol Heart Circ Physiol 2008;295:H2308-14.

19. Penn AH, Schmid-Schonbein GW. Severe intestinal ischemia can trigger cardiovascular collapse and sudden death via a parasympathetic mechanism. Shock 2011;36:251-62.

20. Koksoy C, Kuzu MA, Ergun H, Demirpence E, Zulfikaroglu B. Intestinal ischemia and reperfusion impairs vasomotor functions of pulmonary vascular bed. Ann Surg 2000;231:105-11.

21. Qu B, Xin GR, Zhao LX et al. Testing stem cell therapy in a rat model of inflammatory bowel disease: role of bone marrow stem cells and stem cell factor in mucosal regeneration. PLoS One 2014;9:e107891.

22. Devine SM, Bartholomew AM, Mahmud N et al. Mesenchymal stem cells are capable of homing to the bone marrow of non-human primates following systemic infusion. Experimental hematology 2001;29:244-55.

23. Liu K, Ji K, Guo L et al. Mesenchymal stem cells rescue injured endothelial cells in an in vitro ischemia-reperfusion model via tunneling nanotube like structure-mediated mitochondrial transfer. Microvasc Res 2014;92:10-8.

24. Cho YM, Kim JH, Kim M et al. Mesenchymal stem cells transfer mitochondria to the cells with virtually no mitochondrial function but not with pathogenic mtDNA mutations. PLoS One 2012;7:e32778.

25. Brown DA, Sabbah HN, Shaikh SR. Mitochondrial inner membrane lipids and proteins as targets for decreasing cardiac ischemia/reperfusion injury. Pharmacol Ther 2013;140:258-66.

26. Weil BR, Markel TA, Herrmann JL, Abarbanell AM, Meldrum DR. Mesenchymal stem cells enhance the viability and proliferation of human fetal intestinal epithelial cells following hypoxic injury via paracrine mechanisms. Surgery 2009;146:190-7. 
27. Spinella F, Caprara V, Cianfrocca R et al. The interplay between hypoxia, endothelial and melanoma cells regulates vascularization and cell motility through endothelin-1 and vascular endothelial growth factor. Carcinogenesis 2014;35:840-8.

28. Shi L, Wang L, Wang B et al. Regulatory mechanisms of betacellulin in CXCL8 production from lung cancer cells. J Transl Med 2014;12:70.

29. Crafts TD, Jensen AR, Blocher-Smith EC, Markel TA. Vascular endothelial growth factor: Therapeutic possibilities and challenges for the treatment of ischemia. Cytokine 2014.

30. Bartholomew A, Sturgeon C, Siatskas M et al. Mesenchymal stem cells suppress lymphocyte proliferation in vitro and prolong skin graft survival in vivo. Experimental hematology 2002;30:42-8.

31. Lin CS, Lin G, Lue TF. Allogeneic and xenogeneic transplantation of adipose-derived stem cells in immunocompetent recipients without immunosuppressants. Stem cells and development 2012;21:2770-8.

32. Pigott JH, Ishihara A, Wellman ML, Russell DS, Bertone AL. Investigation of the immune response to autologous, allogeneic, and xenogeneic mesenchymal stem cells after intraarticular injection in horses. Veterinary immunology and immunopathology 2013;156:99106.

33. Leveque X, Mathieux E, Nerriere-Daguin $\vee$ et al. Local control of the host immune response performed with mesenchymal stem cells: perspectives for functional intracerebral xenotransplantation. Journal of cellular and molecular medicine 2015;19:124-34. 